\title{
PARAMETRIC OPTIMIZATION OF AMPHIBIOUS AIRCRAFT PROFILE SKETCH
}

\section{P. A. SVECHNIKOV, M. A. NIKITIN, N. A. KHARALDIN, A. V. STEPANOV, M. V. ALESHIN \& A. I. BOROVKOV}

Automotive Department, Peter the Great St.Petersburg Polytechnic University Engineering Center "ComputerAided Engineering Center", St. Petersburg, Russia

Automotive Department, Peter the Great St.Petersburg Polytechnic University Engineering Center "ComputerAided Engineering Center", St. Petersburg, Russia

Automotive Department, Peter the Great St.Petersburg Polytechnic University Engineering Center "ComputerAided Engineering Center”, St. Petersburg, Russia

Automotive Department, Peter the Great St.Petersburg Polytechnic University Engineering Center "ComputerAided Engineering Center", St. Petersburg, Russia

Automotive Department, Peter the Great St.Petersburg Polytechnic University Engineering Center "ComputerAided Engineering Center”, St. Petersburg, Russia

Professor, innovative projects vice-rector of Peter the Great St.Petersburg, Polytechnic University, St. Petersburg, Russia

\section{ABSTRACT}

The article is about the search of optimal fuselage shape of an amphibious aircraft in terms of aerodynamic characteristics. In order to find the optimal fuselage shape we used the parametric optimization method.

This article proposes an approach that allows you to solve a wide range of engineering problems in finding the appropriate form of an aircraft. In order to check the correctness of the proposed approach to find the optimal fuselage shape of an amphibious aircraft wing parametric optimization was carried out.

The simulation of the aircraft airfoil geometry and the amphibious aircraft fuselage was carried out in the SolidWorks. As a result of aircraft wing optimization one of the possible forms coincided with the Mynk-6 airfoil-shaped profile. The aerodynamic characteristics of the wing were defined using the finite volume method in the ANSYS software package. The found values coincided with the theoretical data, which indicates the correctness of the boundary conditions and the optimization technique. Additionally, the converging of the solution of the block-structured and tetrahedral finite element model was tested.

Using a similar method of parametric optimization with both ANSYS and IOSO the appropriate fuselage shape of the amphibious aircraft is obtained. The optimum fuselage shape has significantly better aerodynamic characteristics compared with a non-optimized model.

KEYWORDS: Parametric Optimization, Flowing of Fuselage Profile, Aerodynamic Characteristics of the Wing, Optimization of Aerodynamic Characteristics, Aerodynamic Calculations, Amphibious Aircraft, Advanced Production Technologies

Received: Jun 09, 2020; Accepted: Jun 29, 2020; Published: Sep 25, 2020; Paper Id.: IJMPERDJUN20201415

\section{INTRODUCTION}

A seaplane is an aircraft designed to take off and land upon water. Currently seaplanes are actively used where the construction of aerodrome is difficult, but there are water ponds where the seaplane operation is possible. Such territories include a number of countries located in taiga and tundra, island states with a large number of rivers and lakes, their small territories do not allow the construction of aerodromes. 
Now a days we have a large number of seaplane models, most of them are floatplanes. Such seaplanes look like a land-based aircraft with one, two or more floats in order to maneuver and park upon water.

Most amphibious air crafts are a flying boat or a floatplane with a land landing gear. The disadvantage of an amphibious aircraft is the uncomfortable cabin and it also has poor aerodynamic characteristics comparing with a similar engine aircraft due to its aerodynamic and hydrodynamic shape.

Thus it is necessary to find the optimal shape of an amphibious aircraft to improve its aerodynamic characteristics.

In this research we used a package of computer design and engineering calculations software - CAD / CAE (Computer Aided Design / Computer Aided Engineering) in order to find the optimal shape of an amphibious aircraft. CAD allows you to create 3D objects and drawings, and also automates the process of design and staging [14].

CML- Bench ${ }^{\mathrm{TM}}$ digital platform (digital platform for digital twins development, their manufacturing production processes, activities managing system in the field of digital design, mathematical modeling and computer engineering) was used in performing engineering calculations, processing and visualization of results. Digital platform CML-Bench ${ }^{\mathrm{TM}}$ is developed by National Technology Initiative Center for Advanced Manufacturing Technologies.

Modern CAE allow you to calculate accurately the technical and operational characteristics of the final product [9].

\section{MAIN PART}

\subsection{Wing Geometry}

To ensure stability and good handling of the aircraft it is necessary to take into account the forces magnitude and other forces influencing on the aircraft. These forces determine the aerodynamics of the aircraft.

Aircraft aero dynamic characteristics are the main indicators of the air impact on the aircraft. These characteristics are determined by the aircraft geometry - the geometric parameters of the wing and fuselage shape.

To verify the correctness of the method of finding the optimal fuselage shape of an amphibious aircraft, as well as the objectivity of the results, we consider a similar problem using the example of the aircraft wing shape.

Wing shape is a wing section by a plane parallel to the original plane of symmetry of the aircraft. The main characteristics that describe the wing geometry are chord, thickness ratio, centerline camber and maximum thickness point. All this data determines the geometry, which means it has a direct effect on the wing drag, ascentional force and the place of transition of the streamlined airflow of the boundary layer to turbulent.

The wing in the air stream changes the streamlines of the ambient air. The speed near the wing changes, which leads to a change in pressure at various points on the surface of the aircraft wing. The air flow rate increases on the wing upper surface, which causes a static pressure decrease. According to Bernoulli's principle the air flow meets an obstacle in front of the wing and on the lower surface, as a result there is a decrease in the flow velocity and an increase in static pressure. Aerodynamic force R appears in conjunction with the nonregular distribution of static pressure over the wing surface. The total aerodynamic force of the wing is the sum of the pressure forces and the tangent friction forces acting on the wing surface. We can see a vector diagram of the pressure distribution over the wing shape on Figure 1. [1]

The aerodynamic force R_A usually consists of two components: the drag force and the lifting force.

The drag force X_A is the projection of R_A on the X axis. It is directed along the approach flow velocity vector, thereby preventing the possible movement of the wing. 
Lifting force $Y \_A$ is the projection of $\mathrm{R} \_A$ on the $\mathrm{Y}$ axis. It is directed opposite to gravitation force. It allows the aircraft to take off and pull up.

Aerodynamic forces are determined by dimensionless Figures: airfoil lift and aerodynamic drag. Aerodynamic wing characteristics are judged according to the dependence graph of the dimensionless Figures of aerodynamic forces on the angle of attack.

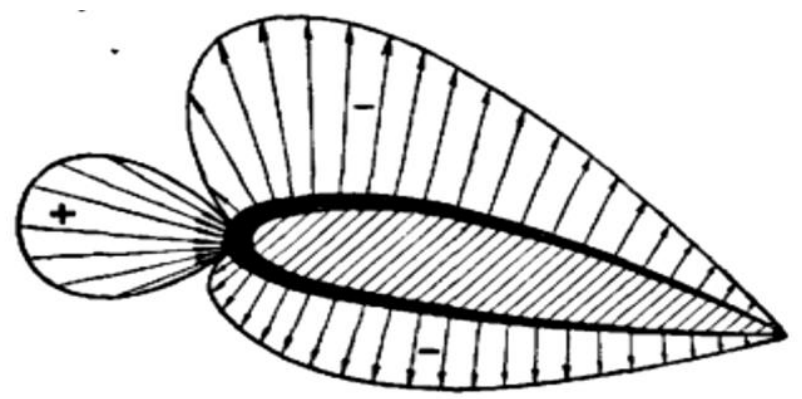

Figure 1: Pressure Distribution Over The Wing Shape.

\subsection{Wing Shape Model Design}

An arbitrary wing shape was proposed to make a comparative analysis (Figure 2). Model geometry preparation was carried out in the SolidWorks. The wing shape is modeled by geometric primitives - directed lines connected by tangents. The directed lines defining the wing leading edge are not being changed. While designing the shape this restriction was introduced so that in the process of parametric optimization, the result of one of the iterations was a geometry that coincided with one of the Mynk series aircraft profiles. In the picture those geometric points that can change their position are highlighted in red squares.

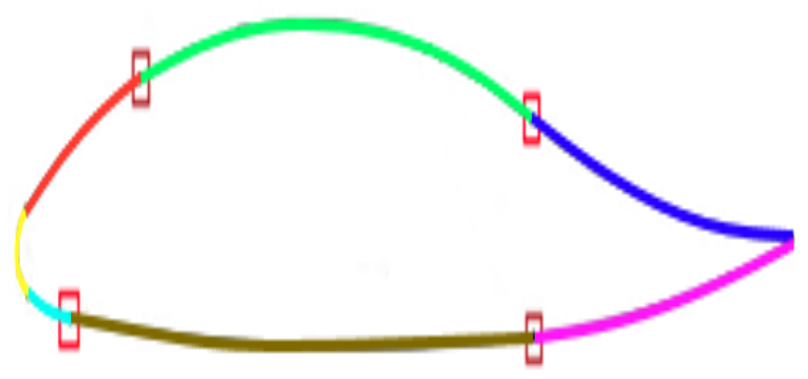

Figure 2: Wing Shape Geometry.

\subsection{Amphibious Aircraft Fuselage Model Design}

The main goal of this article is to get the optimal fuselage shape of an amphibious aircraft using the parametric optimization method. So, in order to fulfil the task an arbitrary fuselage model of an amphibious aircraft was chosen.

The aircraft fuselage is defined by two main sketches shown in Figure 3.

Figure 3 shows the geometry of the model takes into account technological limitations: the thickness of aircraft conical after body, the height of the cockpit and the transparency angle are fixed.

The thickness of the conical afterbody is $60 \mathrm{~mm}$, this value is due to the location of the handle on the tail, which should be no more than $60 \mathrm{~mm}$ in diameter to ensure a comfortable grip. Cabin height is $1350 \mathrm{~mm}$. The transparency angle was selected based on the results of optimization. 
The first aircraft sketch is the curves gained by cutting the fuselage model with the plane of aircraft symmetry (Figure 4). The sketch is modeled in the SolidWorks using curves and arcs connected tangentially to each other. The choice of curves is due to the fact that modeling a sketch only with arcs entails a strong restriction on the variation of the geometric parameters that describe the geometry of the sketch. This restriction is especially observed in the front of the fuselage, so it was decided to model some parts of the profile using curves of the second and higher orders. Higher order curves allow you to define more detailed sketch in connection with advanced interpretations.

Figure 4 shows the sketch of the second profile is a set of curves and arcs obtained by slicing the fuselage with the aircraft base plane, as well as a plane perpendicular to the base plane (Figure 5). Curves describe only fuselage nose, the remaining parts are defined by arcs connected by a tangent. While solving the problem half the sketch is considered because the second part is symmetrical. Taking symmetry into account allows us to halve the computational space. This makes it possible to significantly reduce the time of solving the problem by reducing the number of final volumes in the calculation model.

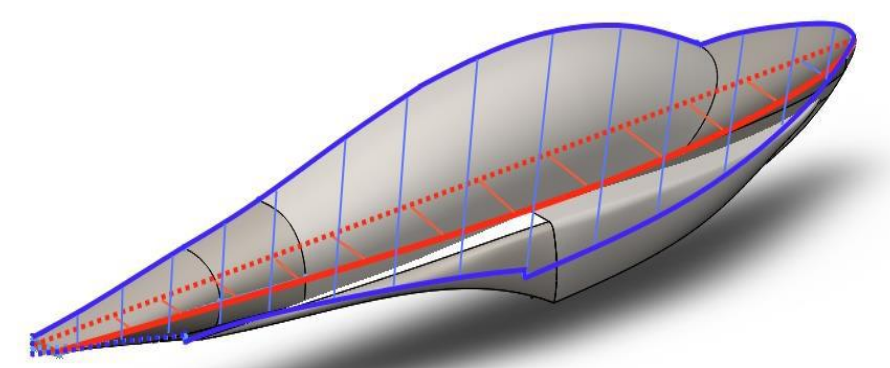

Figure 3: Aircraft Fuselage Profiles.

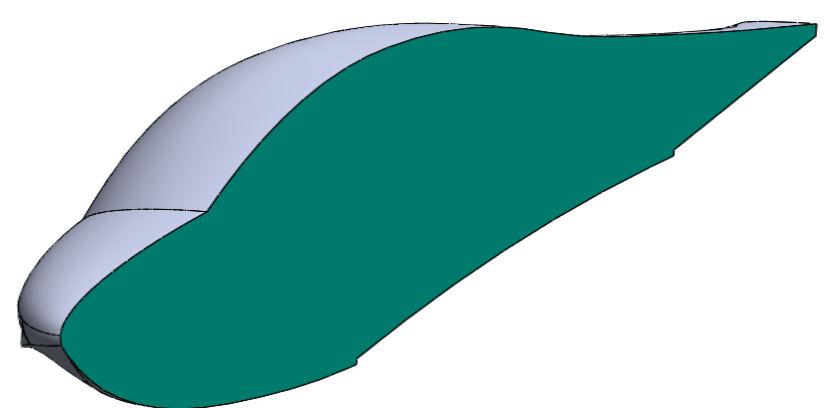

Figure 4: Fuselage slice. Sketch 1.

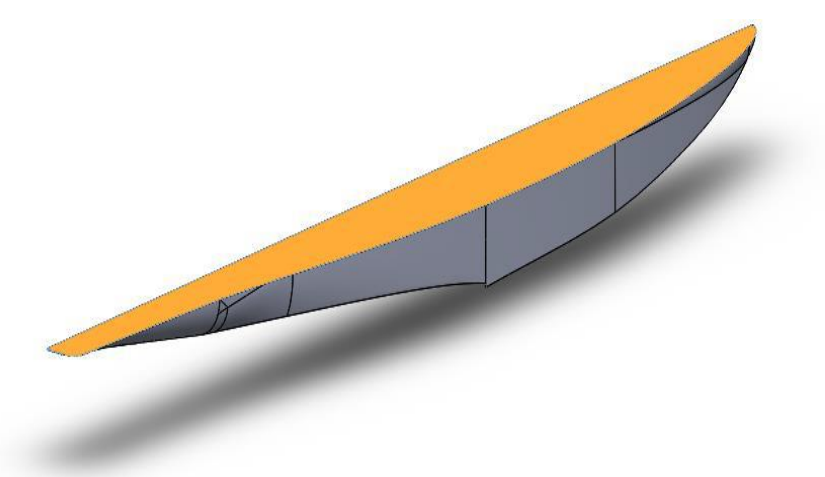

Figure 5: Fuselage Slice. Sketch 2. 


\section{METHODS}

\subsection{Parametric Wing Optimization}

Based on the geometric model a back-end volume mesh is constructed. The airspace region near the wing is simulated. Mesh generation is done in the Ansys ICEM. The geometry is divided into blocks to build a block-structured grid. In addition, grid clustering is set at the wing shape boundary to take into account the boundary layer [10].

The use of unstructured grids significantly increases the resource capacity of the calculation, and as a result the time it takes to solve the problem. [2] The time difference is especially obvious when we deal with the optimization, when there is a huge number of solutions to the same problem with different geometry. In this article the convergence in the number of finite elements is carried out.

To fulfil the task a pseudo-two-dimensional model is simulated. The constructed back-end volume mesh contains 125,184 finite volumes and 82,468 nodes (Figure 6).

Figure 6 shows When solving computational fluid dynamics (CFD) problems the air is conducted to the intake with a $33 \mathrm{~m}$ / s speed. This speed was chosen for comparison with the results gained by aerodynamic airfoil drainage in the Central Institute of Aerohydrodynamics and Moscow Aviation Institute laboratory. Wing incidence is chosen randomly and is $1.5^{\circ}$. There is a condition of zero static pressure to the exit, which is the reference value.

The computational space with boundary conditions is shown in Figure 7. The arrows in the Figure indicate the direction of air flow.

Figure 7 Shows Air was taken as a working substance at a temperature of $\mathrm{T}=25^{\circ} \mathrm{C}$. We chose the turbulence model SST (shear stress transport) for the task; this model more accurately simulates takeoff from faired surface [7].

The Reynolds number in the topic is $\mathrm{Re}=3600$ 000. Totals records file has the output data: dimensionless characteristics of ascentional force and drag component. The calculation is stationary. The calculations were performed using the supercomputer of the center "Polytechnic" SPbPU.

To gain an optimal wing shape with given geometric constraints, the estimated finite-volume part and the solutions obtained are transferred to the IOSO, where parametric optimization is performed [11].

The steps for obtaining the optimization result in the IOSO package are shown in Figure 8. In performance enhancer, the geometry and finite-volume mesh are automatically rebuilt and the aerodynamic problem is solved until the optimization goal is achieved.

Figure 8 shows the optimization criterion is the output parameters of the solution - dimensionless characterisics of ascentional force and drag component. The goal of optimization is to maximize the lift force coefficient and minimize the drag force at a given speed. 

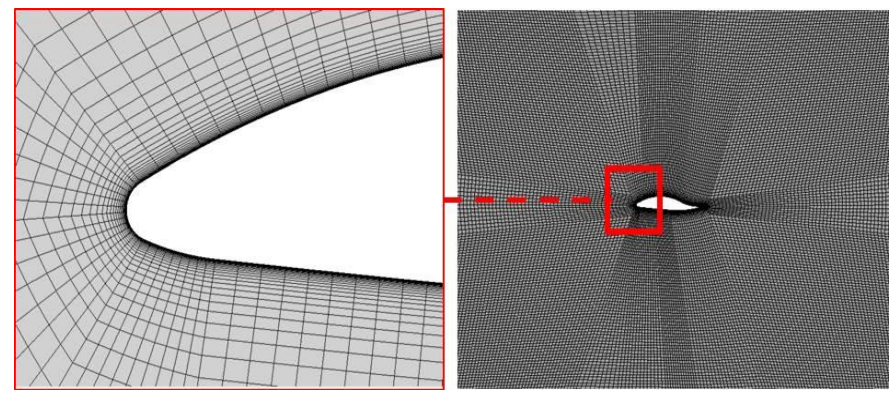

Figure 6: Prediction Model Back-End Volume Mesh.

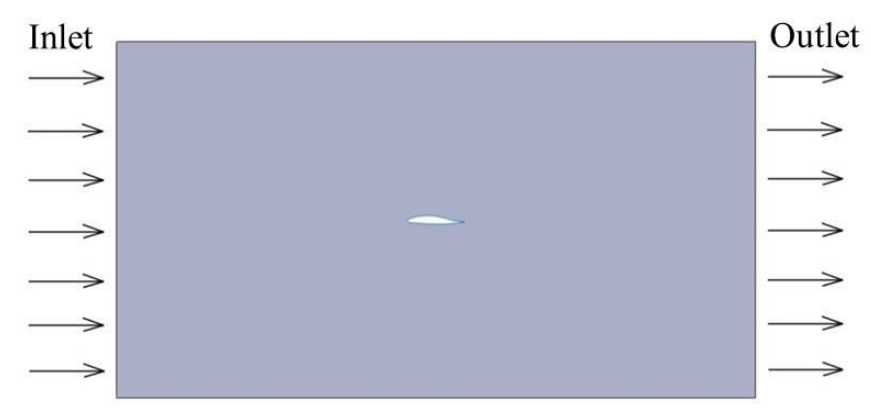

Figure 7: The Computational Space

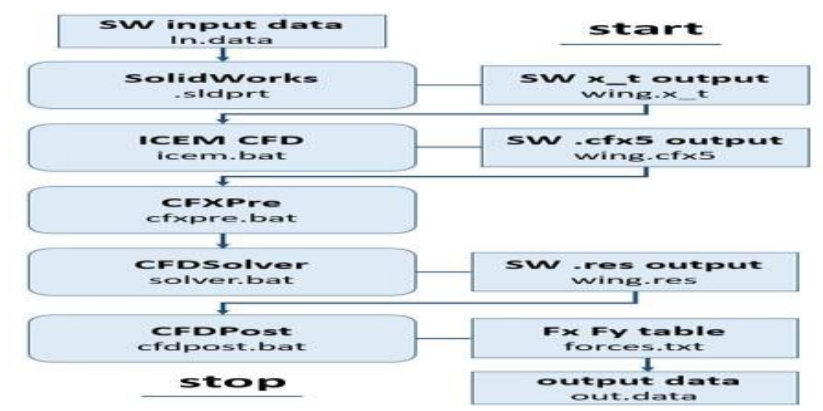

Figure 8: Project Design in IOSO.

\subsection{A Finite-Volume Model Constructing Methods Comparison.}

Parametric optimization involves multiple changing of the model geometry and the aerodynamic task solutions. This process is resourceintensive [12]. The resourceintensity of the task is mainly determined by the number of finite-volumes that are contained in the model. Using tetrahedral elements allows you to create a finite-volume model quickly, in terms of engineering time, because this method does not require the use of additional functionality of grid construction; the main part of the construction is automatic.

To build a tetrahedral network, the original geometry was divided into shell elements of the triangle type. An additional density of network thickening on possible vortex trajectory is set to achieve more accurate results. Additionally, the boundary layer near the wing shape is taken into account by constructing a prismatic layer[8]. Subsequently, a finitevolume tetrahedral network was constructed on the basis of two-dimensional elements, which simulates a pseudo-twodimensional task. An example of constructing such a finite-volume model is presented in Figure 9.

Figure 9 shows for comparison, a block-structured grid was built on a similar computational space. The construction of such a grid is possible if the model geometry is divided into blocks, and accumulation is set at the wing shape boundary to account the boundary layer. Creating such a net is a more complex process and takes more engineering time than 
tetrahedral one, however, a macro in ICEM CFD allows you to rebuild a finite-volume model automatically, you need to build a block-structured net only once. Such a model is presented in the previous part of the article.

12 calculations were made, there were 6 solutions for each type of elements with different amounts of elements. The task setting for all calculations is identical.

The convergence result is shown in Figure 10. We can see that it is more reasonable to use a block-structured grid. Despite the fact that building a block-structured net is a more complex process and it takes more time than a tetrahedral net does, however, for a more reliable solution of one problem fewer final volumes are required than with a tetrahedral net, which means that the model will be less resource-intensive, which in total gives a greater gain in time for solving the problem.

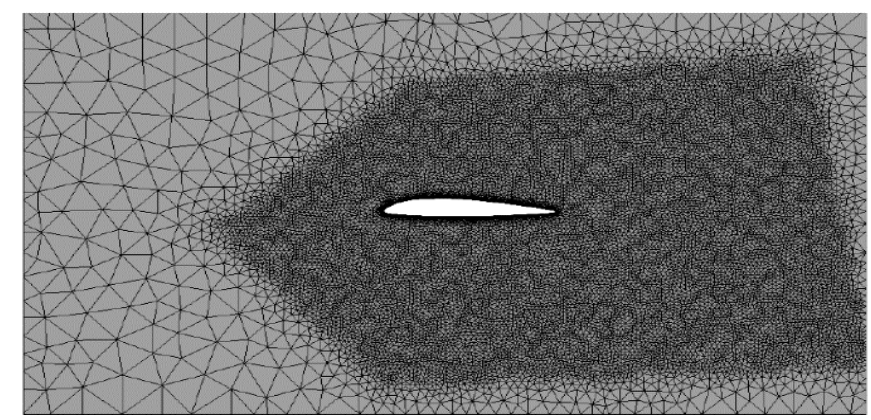

Figure 9: A Finite-Volume Model Constructed With TETRA Type Elements.

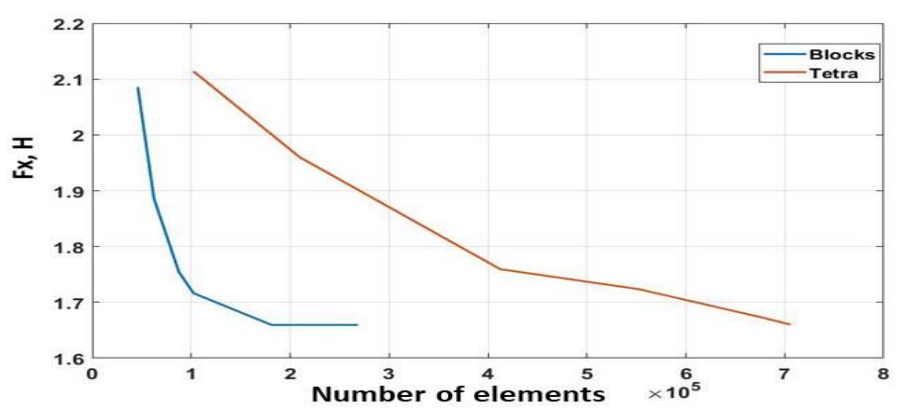

Figure 10: The Convergence of the Solution Depending on the Type of Finite Volumes.

\subsection{Parametric Optimization of the Aircraft Fuselage}

Based on the solution found for the optimal wing airfoil shape, we set a similar task to find the optimal shape of the aircraft fuselage.

To find the optimal shape of an amphibious aircraft fuselage, a finite-volume net was simulated, based on which the computational space of the sketch model 1 was created.

A finite volume model of the computational space is shown in Figure 11. The grid generation is done in the Ansys ICEM. The geometry is divided into blocks to build a block-structured grid. Additionally, grid clustering at the fuselage profile boundary is specified to take into account the boundary layer near this region.

Figure 11 shows the constructed finite-volume mesh has $354636 \mathrm{KO}, 234460$ nodes.

The input air flow is simulated with a $70 \mathrm{~m} / \mathrm{s}$ speed, which corresponds to the cruising air speed of this type of aircraft. The incidence angle is $1.5^{\circ}$ and the model output condition of 0 static pressure were set similarly to the design case 
with the aerodynamic wing shape. The arrows on Figure 12 show the direction of air flow. Both the input stream speed and the incidence angle are chosen in such a way as to simulate the cruise rating of an amphibious aircraft.

Figure 12 shows Air was taken as a working substance at $\mathrm{T}=25^{\circ} \mathrm{C}$. A SST turbulence model was chosen for the task [13]. Totals records file has the output data: characteristics of ascentional force and drag component. The calculation is stationary. The design is made in Ansys CFX.

The optimization criterion is the output characteristics of ascentional force and drag component. The goal of optimization is to maximize the ascentional force coefficient and minimize the drag component at a given speed.

In a similar way to this the sketch 2 computational space was constructed (Figure 13). The input air flow value is $70 \mathrm{~m} / \mathrm{s}$. The model output condition of 0 static pressure is set. On the boundary lying in the plane of symmetry of the aircraft the symmetry condition is set. The arrows indicate the direction of air flow.

Figure 13 shows Air was taken as a working substance at $\mathrm{T}=25^{\circ} \mathrm{C}$. A SST turbulence model was chosen for the task. As the output data we have a drag component. The ascentional force is not considered since the profile is blown in a plane perpendicular to this force.

To get the most reliable result the geometry is divided into blocks for constructing a block-structured grid. Additionally, grid clustering at the fuselage profile boundary is specified to take into account the boundary layer near this region.

The constructed finite-volume mesh of the geometric model is shown in Figure 14.

Figure 14 shows the constructed finite-volume mesh has 92379 KO, 60638 nodes.

When being constructed the finite-volume mesh is imported into the IOSO software for parametric optimization. The optimization criterion is the drag component. The goal of optimization is to minimize the drag component at a given speed.

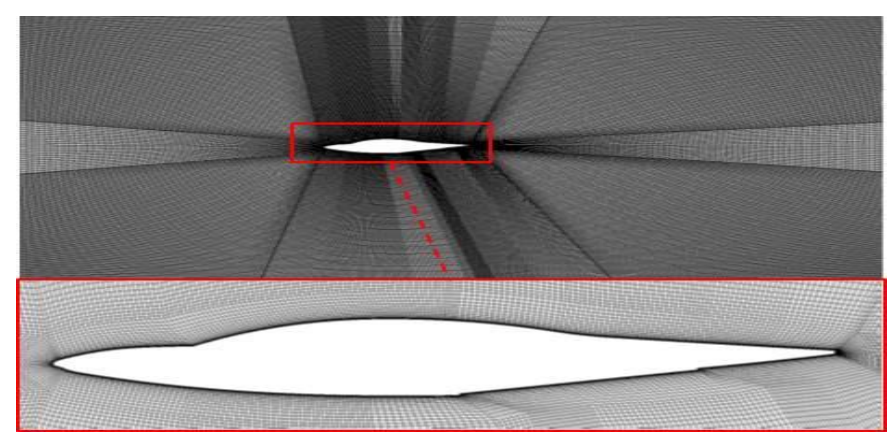

Figure 11:The Finite-Volume Mesh and Its Details.

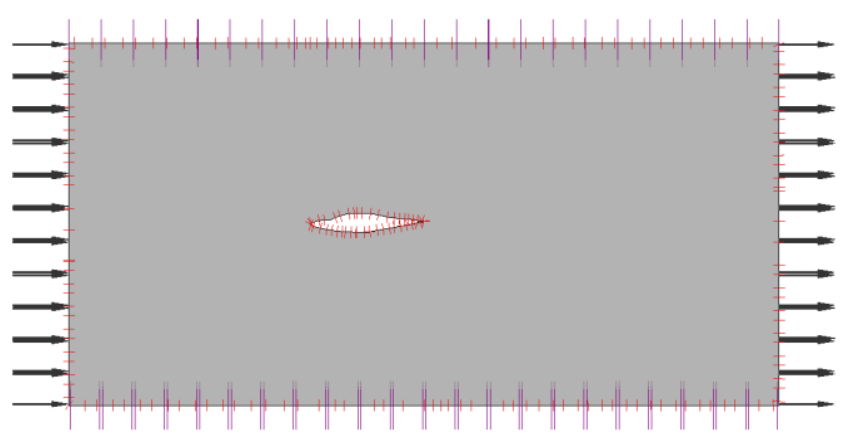

Figure 12: Computational Space. Sketch 1. 


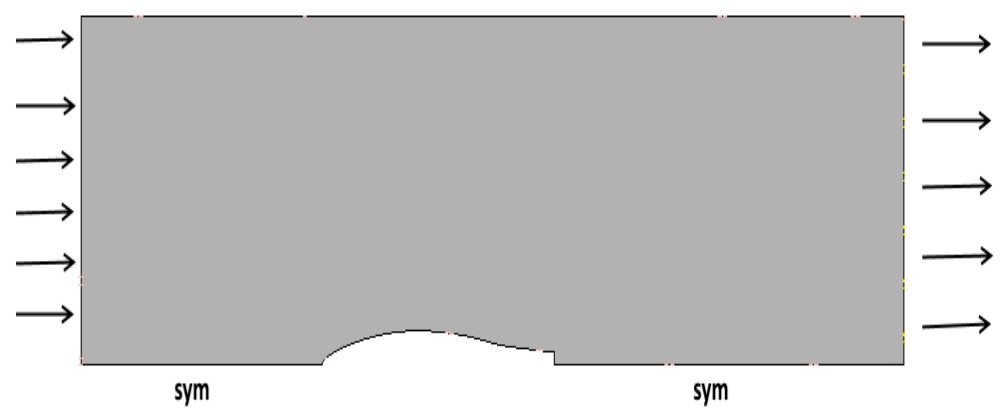

Figure 13: Computational Space. Sketch 2.

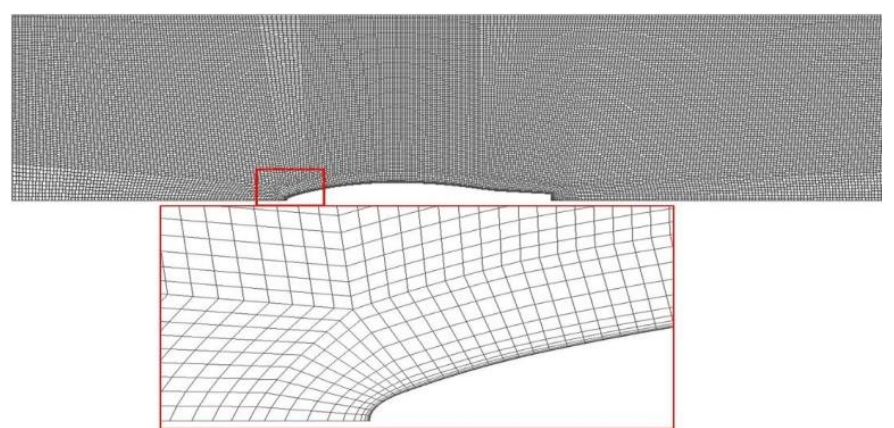

Figure 14: The Finite-Volume Mesh And Its Details.

\section{RESULTS}

\subsection{The Aircraft Wing Shape Optimization Result}

The search history of wing shape

optimization is presented as a graph of Cy vs Cx (Figure 15). The highlighted point No. 1 corresponds to the aerodynamic characteristics of the Mynk-6 aviation profile: $\mathrm{Cx}=0.00958, \mathrm{Cy}=0.1257$ (Table 1). [4] The maximum deviation of experimental values is $1.24 \%$, which can be considered as a reliable result.

The wing shape geometry received as a result of optimization also coincides with the Mynk-6 profile. Additionally, calculations for different incidence angles were performed with this profile. A connection for each aerodynamic dimensionless coefficient is constructed and a comparison is made with experimental data (Figure 16). The values of the dimensionless drag coefficient $\mathrm{Cx}$ have a very small deviation from the table values.

Figure 16 shows Point 2 in is the most optimal profile shape, where the Cy parameter is the maximum one. Dimensionless drag coefficients $\mathrm{Cx}$ is 0.0098 , lift force coefficients $\mathrm{Cy}$ is 0.1487 . Below is the optimal wing shape under the given restrictions in comparison with the Mynk-6 profile (Figure 17).

Figure 18 shows the air flow distribution for the Mynk-6 profile, Figure 19 shows the air flow distribution for a profile found by optimization.

Figure 19 shows the presented speed distribution graphs we can see that the stream velocity under the wing of the optimized model is lower than the stream velocity under the Mynk-6 wing, and above wing even more. Bernoulli equationsays that as a result of circulation the pressure under the wing of the optimized profile will be more, and above the wing less comparing to Mynk-6 [15]. The pressure difference created can be seen in a lifting force value change. 


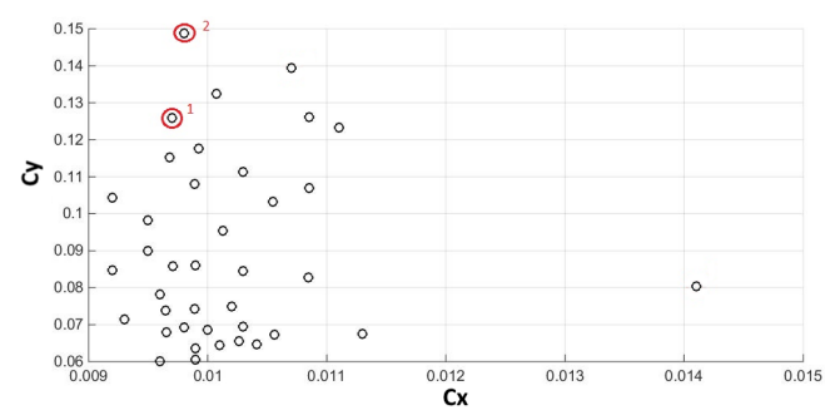

Figure 15: Optimization Search History.

Table 1: Mynk-6 Profile Aerodynamic Characteristics

\begin{tabular}{|c|c|c|}
\hline \multicolumn{3}{|c|}{ Aerodynamic characteristics } \\
\hline $\boldsymbol{\alpha}^{\mathbf{0}}$ & $\mathbf{C y}$ & $\mathbf{C x}$ \\
\hline 0 & 0.016 & 0.0008 \\
\hline 1.5 & 0.126 & 0.0097 \\
\hline 3 & 0.237 & 0.0111 \\
\hline 4.5 & 0.340 & 0.0147 \\
\hline 6 & 0.456 & 0.0212 \\
\hline
\end{tabular}

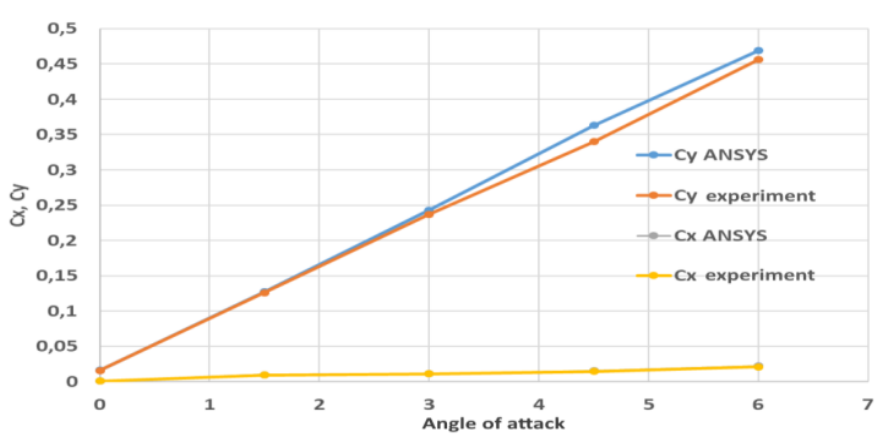

Figure 16: Cx and Cy Results Comparison At Different Incidence Angles.

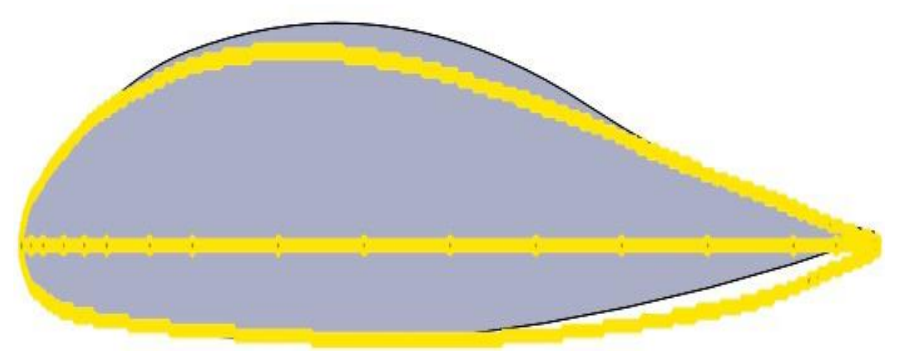

Figure 17: Mynk-6 Wing Shape (In Yellow) and Optimization Result.

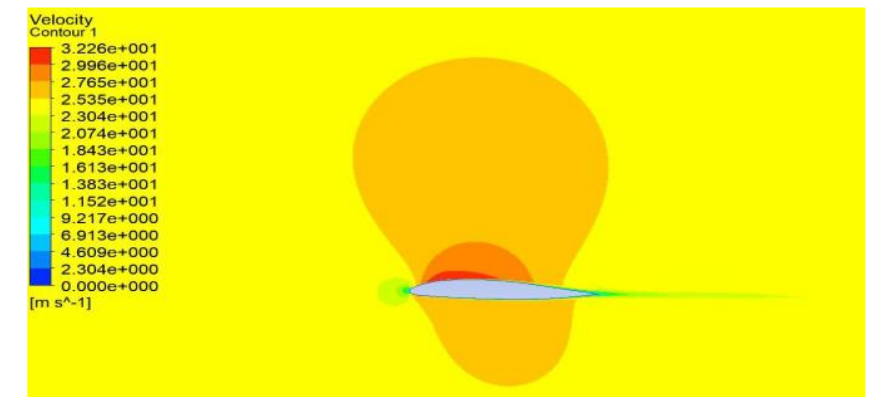

Figure 18: Air Speed Near The Mynk-6 Profile. 


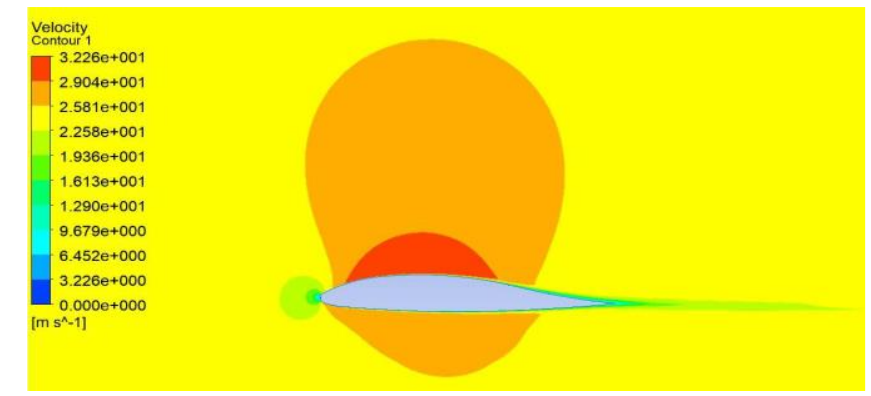

Figure 19: Air Speed Near the Optimized Wing.

\subsection{Model 1 Sketch Optimization Result}

Model 1 sketch parametric optimization was carried out in the IOSO. The time needed for one task was 10 minutes. The iteration convergence showed that after 179 iterations the deviation of results is acceptable. The solutions search history is presented in Figure 20.

In Figure 20 we can see the Pareto Front, which characterizes the search for the optimal solution. [5] The main factor of the fuselage profile optimality is the drag component. Thus, the optimization values that we need are located near the horizontal line $\mathrm{Fx}=2 \mathrm{H}$.

Due to the fact that the fuselage nose was modeled by curves, it required additional study, because of particular technological structure of the body. The final model for the first profile is shown in Figure 21.

Drag component and ascentional force are calculated, values of which we can see in Table 2. The drag component decreased by $20 \%$ comparing to the original model, the ascentional force increased by $1.1 \%$. These indicators significantly improve the aerodynamic characteristics of the entire fuselage.

Profile flow of the model is shown in Figure 22.

Detailed visual analysis showed that the main change in geometry occurred in the fore-body (Figure 23). Minor changes occurred near the cockpit, which means that cabin space in the new fuselage model will remain comfortable for the pilot.

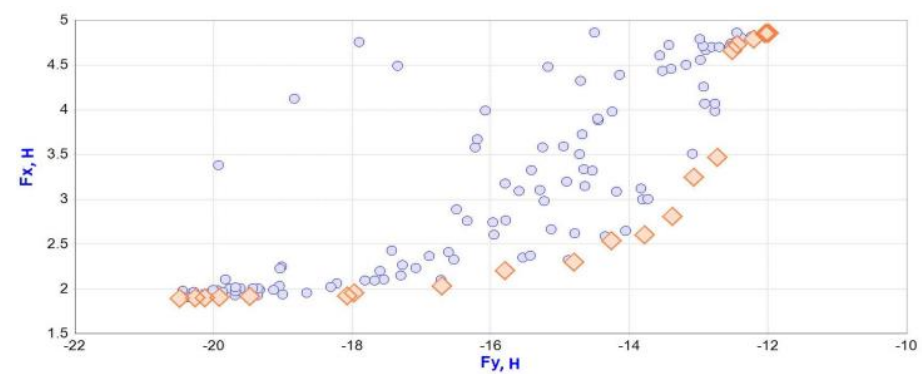

Figure 20: Search History.

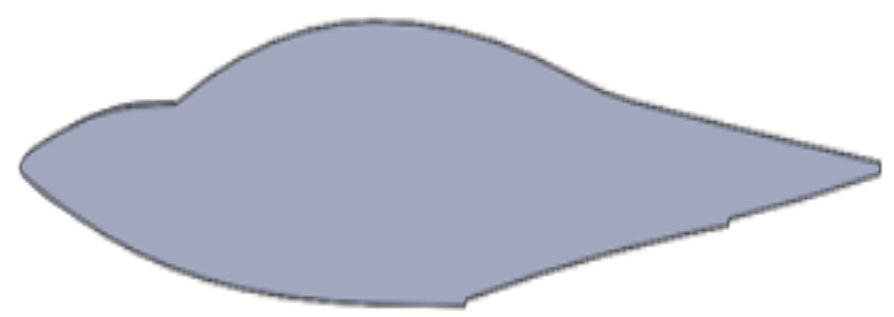

Figure 21: Optimized Model With Processing Limits. 


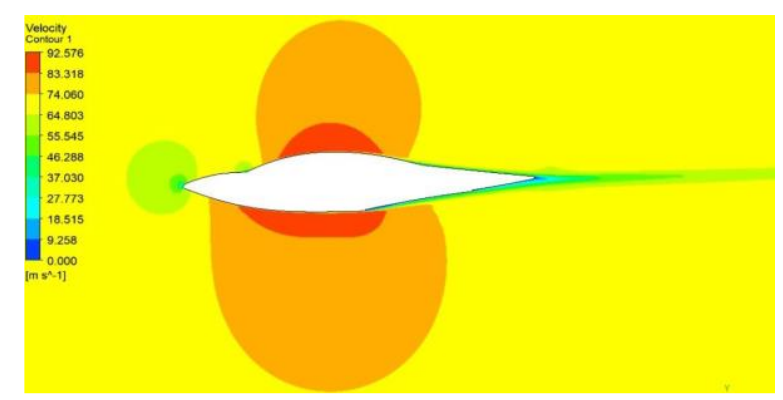

Figure 22: Profile Flow.

Table 2: Optimization Results Comparison

\begin{tabular}{|c|c|c|c|}
\hline \multirow{2}{*}{} & \multicolumn{3}{|c|}{ Aerodynamic Forces } \\
\cline { 2 - 4 } & Original & Optimized & Difference \\
\hline Fx, N & 2.437 & 1.945 & $20.2 \%$ \\
\hline Fy, N & -17.961 & -17.761 & $1.1 \%$ \\
\hline
\end{tabular}

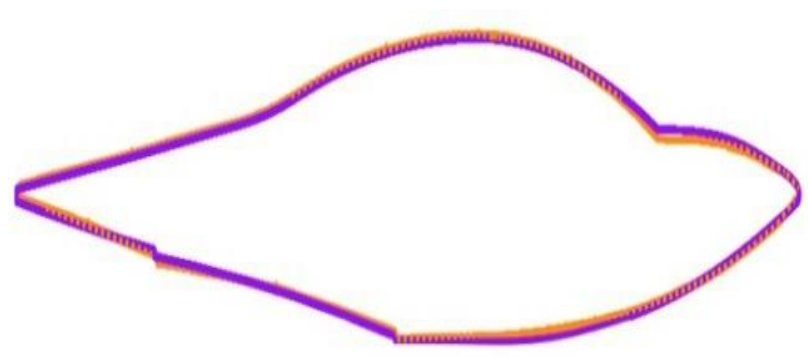

Figure 23: Original Model (Orange) and Optimized Model(Purple) Profiles Comparison.

\subsection{Model 2 Sketch Optimization Result}

Model 2 sketch parametric optimization was carried out in the IOSO by a similar way.

The time needed for one task was 3 minutes. The iteration convergence showed that after 211 iterations the deviation of results is acceptable. The solutions search history is presented in Figure 24.

Figure 24 shows We can see the convergence of the solution on the drag component value $\mathrm{Fx}=1.032 \mathrm{H}$.

The optimization and profile drainage result is shown in Figure 25 and Figure 26 respectively.

Drag component and its comparison is shown in Table 3. The difference from the original geometry is more than $7 \%$.

Table 3 shows We can see geometric changes in the forebody and taper ratio in the middle part (Fig. 27). The width change comparing with the original profile is $18 \mathrm{~mm}$.

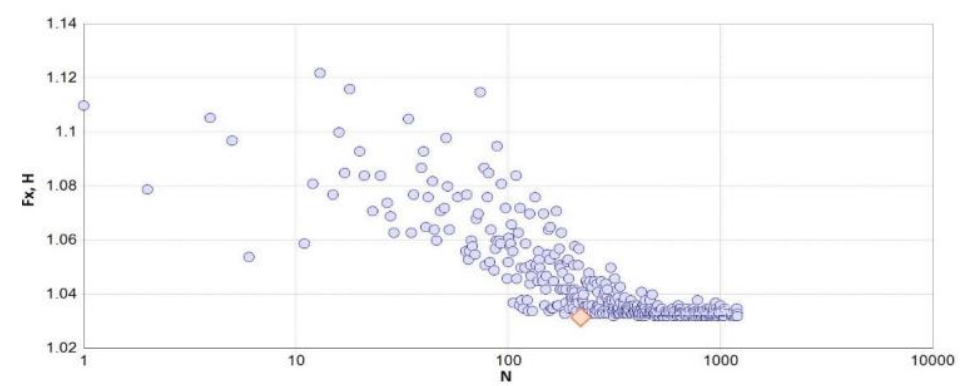

Figure 24: Search History. 


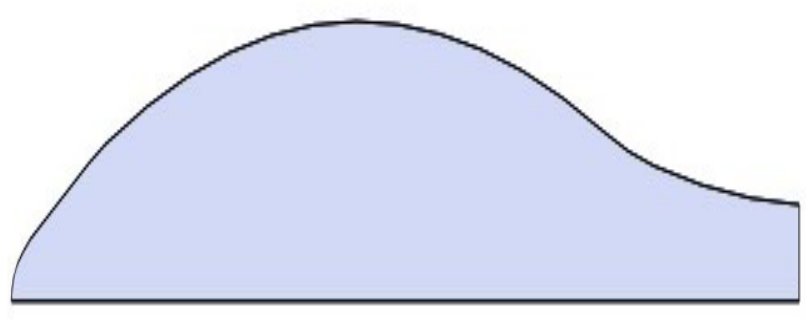

Figure 25: Sketch 2 Optimal Profile.

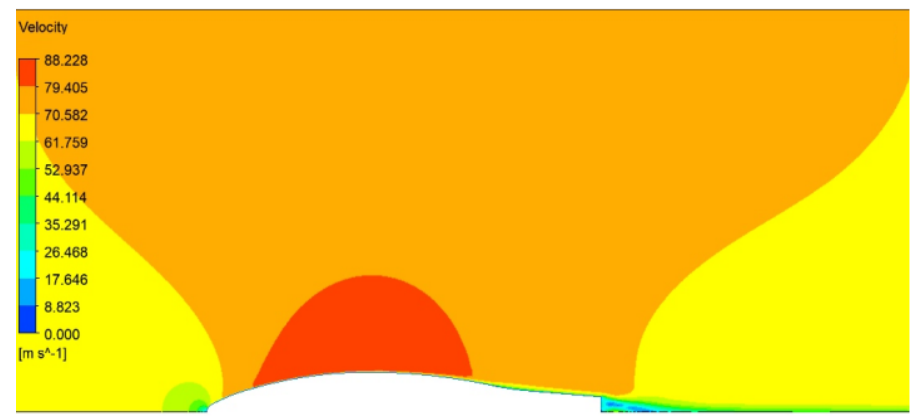

Figure 26: Airfoil Flow.

Table 3: Optimization Result Comparison

\begin{tabular}{|c|c|c|c|}
\hline \multirow{2}{*}{} & \multicolumn{3}{|c|}{ Aerodynamic Forces } \\
\cline { 2 - 4 } & Original & Optimized & Difference \\
\hline Fx, N & 1.110 & 1.032 & $7 \%$ \\
\hline
\end{tabular}

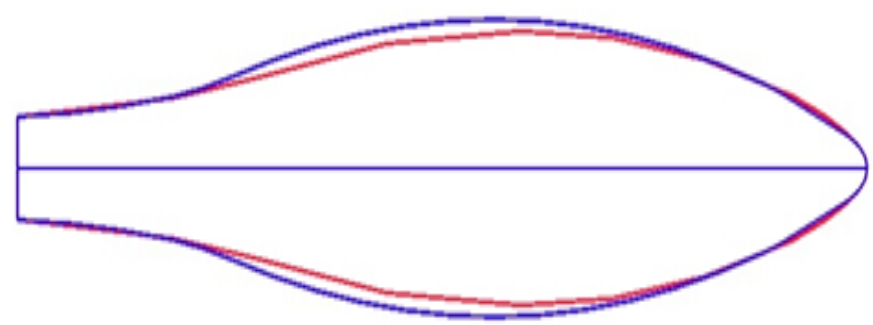

Figure 27: Original Model (Blue) and Optimized Model (Red) Profiles Comparison.

\subsection{3-D model. Results Comparison}

To get a general picture of the optimization result, a three-dimensional model based on optimized profiles was constructed (Figure 28).

Figure 28 shows à visual difference from the original three-dimensional model is seen in many local parts while comparing both models in detail, so it is not provided in this article.

To compare aerodynamic characteristics a calculation of both three-dimensional models was carried out. The air flow distribution is shown in Figure 29 and Figure 30.

As a result of optimization it was possible to reduce the drag component of 3D model by $9 \%$ and increase the as centional force by $6.7 \%$ (Table 4). 


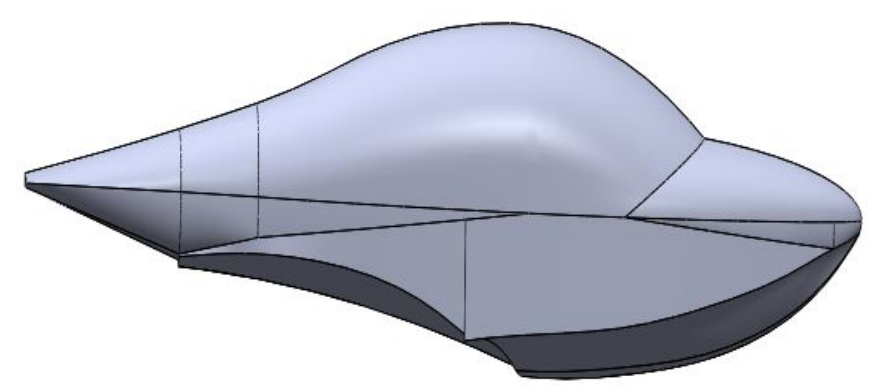

Figure 28: 3-D Model Built Using Optimized Profiles.

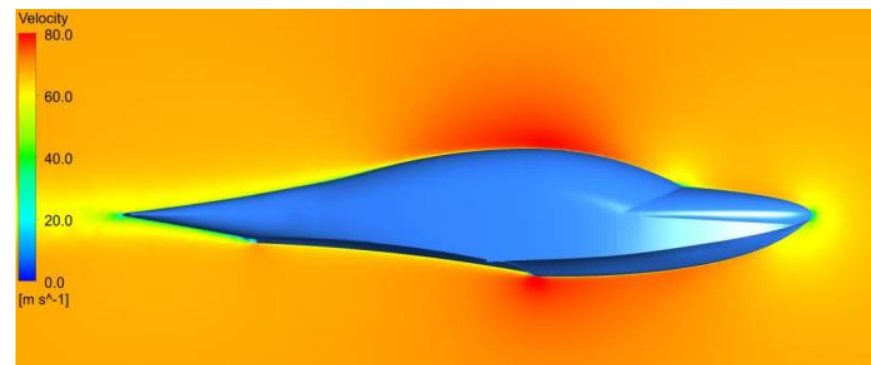

Figure 29: The Air Flow Distribution along the Optimal Model. Profile View 1.

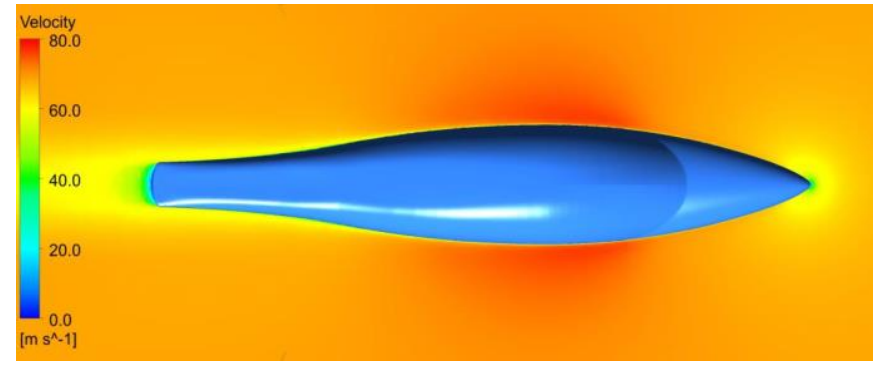

Figure 30: The Air Flow Distribution along the Optimal Model. Profile View 2.

Table 4: Optimization Result Comparison

\begin{tabular}{|c|c|c|c|}
\hline \multirow{2}{*}{} & \multicolumn{3}{|c|}{ Aerodynamic forces } \\
\cline { 2 - 4 } & Original & Optimized & Difference \\
\hline Fx, N & 196.48 & 178.80 & $-9.00 \%$ \\
\hline Fy, N & -67.33 & -62.80 & $6.70 \%$ \\
\hline
\end{tabular}

\section{CONCLUSIONS}

The article is about the search of optimal fuselage shape of an amphibious aircraft. The optimal shape was searched using parametric optimization. The fuselage model was determined by two profiles. Consequently, the task was set for each profile separately and then it was combined into a 3-D model. To verify the correctness of the proposed approach and get a reliable result, a model task was set and verification with experimental data was carried out. Being convinced that the model task data is reliable, this method was also applied to the main task. After setting the boundary conditions and constructing the optimization space, the finite-volume model of two fuselage profiles was imported for parametric optimization. Due to the fact that the fuselage nose was simulated by curves, it required additional engineering study because of technological structure characteristics of fuselage. CFD calculations were made based on the final model to find the aerodynamic characteristics: drag component and ascentional force. As a result of optimization the drag component of 3D model decreased 
by $9 \%$ and the ascentional force increased by $6.7 \%$. Therefore the optimal amphibious aircraft fuselage shape has significantly better aerodynamic characteristics comparing to the non-optimized model.

Based on the obtained results we can say that it is possible to develop the methodology with multicriteria optimization of various fuselage elements at different flight modes, as well as adjusting the model for hydrodynamic forces effects recording. This approach will allow us to develop a model algorithm to work with different types of aircraft.

\section{REFERENCES}

1. V.A. Frolov "Aerodynamic characteristics of the profile and wing": methodical manual, Samara: state aerospace industry, 2007.

2. E.M. Smirnov, D.K. Zaitsev "Finite volume method in application to problems of hydro-gas dyna mics and heat transfer in areas of complex geometry. ", Scientific and technical journal, 2004.

3. M.A.Shcherbakov, B.A.Krylov "Comparative analysis of turbulence models using the scientific code "Fastest-3D" and the commercial package ANSYS CFX.", Branch of NTC named after A. Lyulka of STC " NPO " Saturn».

4. Directory of aviation profiles [Online]. Available: https://kipla.kai.ru/liter/Spravochnic_avia_profiley.pdf(In Rusian).

5. Y. Yorozu, M. Hirano, K. Oka, and Y. Tagawa, "Electron spectroscopy studies on magneto-optical media and plastic substrate interface,” IEEE Transl. J. Magn. Japan, vol. 2, pp. 740-741, August 1987 [Digests 9th Annual Conf. Magnetics Japan, p. 301, 1982].

6. I.N Egorov, G.V.Kretinin, I. A. Leshchenko "Robust design optimization strategy of IOSO technology”, Proc. Fifth World Congress on Computational Mechanics. Vienna. Austria. 2002.

7. A.I Troshin "A semi-empirical turbulence model for describing high-speed mixing layers and jets, not based on the Boussinesq hypothesis", The dissertation on competition of a scientific degree of candidate of physical and mathematical Sciences. -168 p.

8. C. Wilcox “Turbulence modeling for CFD”- DCW Industries, 1993.

9. I. Borovkov "Perspective directions of development of advanced production technologies in Russia" XVII April international scientific conference on problems of development of economy and society. In four books. Book 3. Ed. by E. Yasin, Moscow: HSE, 2017, Pp. 381389.

10. Molchanov A. M., Shcherbakov M. A., Yanyshev D. S., Kuprikov M. Yu., Bykov L. V. "Construction of grids in the problems of aviation and space technology”. Handbook for students; MAI-Moscow, 2013.260 p.

11. I.N. Egorov, G.V. Kretinin, I.A. Leshchenko, S.V. Kuptzov "IOSO Optimization Toolkit - Novel Software to Create Better Design” AIAA paper AIAA-2002-5514, 9th AIAA/ ISSMO Symposium and Exibit on Multidisciplinary Analysis and Optimization, Atlanta, GA, 4-6 September. -2002 .

12. S. Nemirovsky “Complexity of problems and efficiency of optimization methods.” - Moscow: Nauka, 1979. 384 p.

13. W. Rodi “Turbulence Models and Their Application in ydraulics." - Delft, Netherlands: International Association for Hydraulic Research (IAHR), 1980.

14. I. Borovkov, S. F. Burdakov, O. I. Klyavin, M. P. Melnikova, A. A. Mikhailov, A. S. Nemov, V. A. Palmov, E. N. Silina "Computer engineering”- Saint Petersburg: Polytechnic University Publishing house. UN-TA, 2012.93 p.

15. L. G. Lotsyansky “Fluid Dynamics”:Textbookfor universities, Moscow: Drofa, $2003-840$ p. 